\title{
A COMPLETE SYSTEM OF TENSORS OF LINEAR HOMOGENEOUS SECOND-ORDER DIFFERENTIAL EQUATIONS*
}

\author{
BY \\ CLYDE M. CRAMLET
}

\section{INTRODUCTION}

In an article appearing in the American Mathematical Monthly $\dagger$ Lane called attention to the need in projective differential geometry of an absolute differential calculus for forms of higher degree than the quadratic. Such a calculus for forms of arbitrary degree had been published by the writer $\ddagger$ and it seemed that some adaptation to the theory of projective differential geometry would be desirable. However, it appeared more satisfactory to base a tensor theory directly upon the differential equations. Inasmuch as various systems of differential equations are used in the projective theory, a separate tensor theory will need to be constructed for each type. In this paper a study has been made of the differential equations upon which the ruled surface theory is based.

The remarkable simplicity which has been introduced by tensor methods in the theory of Riemannian geometry and of algebraic invariants appears also in this theory. The Wilczynski theory, while general, has had the blemish of containing awkward unsymmetrical sets of equations involving cumbersome numerical constants. And with the improvement in notation there is an accompanying advantage in method, the simple formal tensor processes replacing the earlier ingenious methods.

Some geometric interpretations of the tensors and invariants are given here in which the dependent variables $y^{i}(i=1, \cdots, n)$ are interpreted as nonhomogeneous coördinates of a point. This is in contrast to the convention peculiar to projective differential geometry of choosing a fundamental set of $2 n$ independent solutions $\left(y_{\alpha \mid}^{1}, \cdots, y_{\alpha \mid}^{n}\right), \alpha=1, \cdots, 2 n$, and interpreting these as $n$ points $\left(y_{11}^{i}, \cdots, y_{2 n k}^{i}\right), i=1, \cdots, n$, in a homogeneous space of $2 n-1$ dimensions. An interpretation of our tensors in this latter space will constitute a generalization of Wilczynski's ruled surface theory.

\footnotetext{
* Presented to the Society, December 30, 1930; received by the editors October 22, 1931, and (revised) January 22, 1932.

$\dagger$ E. P. Lane, Present tendencies in projective geometry, American Mathematical Monthly, vol. 37 (1930), pp. 212-216.

$\ddagger$ Annals of Mathematics, (2), vol. 31 (1930), pp. 134-150.
} 
Acknowledgment is due Professor A. F. Carpenter for his interest and helpful criticism in the preparation of this paper

TRANSFORMATIONS ON THE DEPENDENT VARIABLES

1. The most general system of $n$ linear homogeneous differential equations of the second order in $n$ dependent and one independent variable may be solved* for the second derivatives and written

$$
\frac{d^{2} y^{i}}{d x^{2}}+p_{\alpha}^{i} \frac{d y^{\alpha}}{d x}+q_{\alpha}^{i} y^{\alpha}=0
$$

The most general transformations $(n>1)$ that leave these invariant in degree and order are $\dagger$

$$
G: \begin{array}{ll}
(1) & \bar{y}^{2}=\bar{a}_{\alpha}^{i} y^{\alpha}, \\
(2) & \bar{x}=\xi(x),
\end{array}
$$

where the $\bar{a}_{r}^{8}$ and $\xi$ are arbitrary analytic functions of $x$.

A given set of differential equations and all equations obtained from them by these transformations will be called equivalent under the group of transformations. To study the properties common to equations equivalent under the group is to study the invariants, or invariant equations, under the group. Any tensor equation is an invariant equation and from tensors invariants are readily formed; so the first problem will be to find the fundamental set of tensors expressing the equivalence of equations of the form (1:1).

When $\left|\bar{a}_{r}^{s}\right| \neq 0$,

$$
\begin{gathered}
y^{i}=a_{\alpha}^{i} \bar{y}^{\alpha}, \\
\bar{a}_{\alpha}^{i} a_{j}^{\alpha}=a_{\alpha}^{i} \bar{a}_{j}^{\alpha}=\delta_{j}^{i} .
\end{gathered}
$$

From $(1: 3)$

$$
\begin{aligned}
& \frac{d y^{i}}{d x}=a_{\alpha}^{i} \frac{d \bar{y}^{\alpha}}{d x}+\frac{d a_{\alpha}^{i}}{d x} \bar{y}^{\alpha}, \\
& \frac{d^{2} y^{i}}{d x^{2}}=a_{\alpha}^{i} \frac{d^{2} \bar{y}^{\alpha}}{d x^{2}}+2 \frac{d a_{\alpha}^{i}}{d x} \frac{d \bar{y}^{\alpha}}{d x}+\frac{d^{2} a_{\alpha}^{i}}{d x^{2}} \bar{y}^{\alpha} .
\end{aligned}
$$

* In case the determinant of the coefficients of the $d^{2} y^{i} / d x^{2}$ is of rank $r$ the $d^{2} y^{i} / d x^{2}$ may be eliminated from $n-r$ equations. By differentiating these, the equations may be put in the form (1:1).

$\dagger$ Wilczynski, Projective Differential Geometry, p. 14. 
Thus the left member of equation (1:1), subjected to transformations on the dependent variables only, becomes

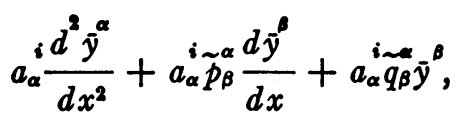

where

$$
\begin{aligned}
& a_{\alpha}^{i} \tilde{p}_{\beta}^{\alpha}=a_{\beta}^{\alpha} p_{\alpha}^{i}+2 \frac{d a_{\beta}^{i}}{d x} \\
& a_{\alpha}^{i \sim \alpha} q_{\beta}^{\alpha}=a_{\beta}^{\alpha} q_{\alpha}^{i}+p_{\alpha}^{i} \frac{d a_{\beta}^{\alpha}}{d x}+\frac{d^{2} a_{\alpha}^{i}}{d x^{2}} .
\end{aligned}
$$

If the left members of equations $(1: 1)$ be denoted by $Y^{i}$ and

$$
\tilde{Y}^{i}=\frac{d^{2} \bar{y}^{i}}{d x^{2}}+\tilde{p}_{\beta}^{i} \frac{d \bar{y}^{\beta}}{d x}+\tilde{q}_{\beta}^{i} \bar{y}^{\beta},
$$

then from $(1: 7)$

$$
Y^{i}=a_{\alpha}^{i} \tilde{Y}^{i} .
$$

Hence equations (1:1) are equivalent to

$$
\frac{d^{2} \bar{y}^{\alpha}}{d x^{2}}+\tilde{p}_{\alpha}^{i} \frac{d \bar{y}^{\alpha}}{d x^{2}}+\tilde{q}_{\alpha}^{i} \bar{y}^{\alpha}=0,
$$

and the relations between the coefficients are given by equations $(1: 8)$ and $(1: 9)$.

Two sets of equations equivalent to these latter but not involving the derivatives of the $a_{r}^{*}$ will be found.

By differentiating (1:8) and eliminating these derivatives from the resulting equations taken in conjunction with $(1: 8)$ and $(1: 9)$, we have

$$
\underset{a_{\beta} u_{\alpha}^{k}}{k} \tilde{u}^{\beta} \underset{u_{\beta}^{k} a_{\alpha}^{\beta}}{k}
$$

where

$$
2 u_{\alpha}^{\beta}=\frac{d p_{\alpha}^{\beta}}{d x}+\frac{1}{2} p_{\alpha}^{\beta} p_{p}^{\beta}-2 q_{\alpha}^{\beta},
$$

with the corresponding definition of $\tilde{u}_{\alpha}^{\beta}$.

By differentiating (1:13) and eliminating the $d a_{\alpha}^{\beta} / d x$ by (1:8), 


$$
\begin{aligned}
a_{\beta}^{k} \bar{u}_{\alpha}^{\prime \beta} & =u_{\beta}^{\prime k} a_{\alpha}^{\beta}, \\
u_{\alpha}^{\prime \beta} & =\frac{d u_{\alpha}^{\beta}}{d x}+\frac{1}{2}\left(p_{\rho}^{\beta} u_{\alpha}^{p}-p_{\alpha}^{p} u_{\rho}^{\beta}\right) .
\end{aligned}
$$

$u_{\alpha}^{\prime \beta}$ will be called the semi-tensor derivative of $u_{\alpha}^{\beta}$ and the process and formula are general.

The semi-tensor $u_{r}^{:}$and its tensor derivatives completely determine a set of equations equivalent to the set $(1: 1)$. Proof of this will be inferred from a more general result in $\$ 6$.

THE TRANSFORMATION ON THE PARAMETER

2. The parameter may be transformed arbitrarily by

$$
\bar{x}=\xi(x) .
$$

If the independent variable is transformed in the left member of the differential equations $(1: 1)$ or $(1: 12)$, that is, either before or after the dependent variables are changed, these quantities transform to $\xi^{\prime 2} \bar{Y}^{i}$,

$$
\begin{aligned}
\bar{Y}^{i} & \equiv \frac{d^{2} \bar{y}^{i}}{d \bar{x}^{2}}+\bar{p}_{\alpha}^{i} \frac{d \bar{y}^{\alpha}}{d \bar{x}}+\bar{q}_{\alpha}^{i} \bar{y}^{\alpha}, \\
\xi^{\prime} & =\frac{d \bar{x}}{d x}=\frac{d \xi}{d x}, \\
\xi^{\prime 2} \bar{p}_{\alpha}^{i} & =\delta_{\alpha}^{i} \xi^{\prime \prime}+\tilde{p}_{\alpha}^{i} \xi^{\prime}, \\
\xi^{\prime 2} \bar{q}_{\alpha}^{i} & =\tilde{q}_{\alpha}^{i} .
\end{aligned}
$$

Hence $(1: 11)$ becomes

$$
Y^{i}=\xi^{\prime 2} a_{\alpha}^{i} \bar{Y}^{\alpha}
$$

Equations (1:1) imply

$$
\frac{d^{2} \bar{y}^{i}}{d \bar{x}^{2}}+\bar{p}_{\alpha}^{i} \frac{d y^{\alpha}}{d \bar{x}}+\bar{q}_{\alpha}^{i} \bar{y}^{\alpha}=0
$$

When the left members of $(1: 8)$ and $(1: 9)$ are substituted in equations $(2: 3)$ and $(2: 4)$, we obtain

$$
\begin{aligned}
& \xi^{\prime 2} \bar{p}_{\alpha}^{i}=\delta_{\alpha}^{i} \xi^{\prime \prime}+\xi^{\prime} \bar{a}_{\sigma}^{i \beta} a_{\alpha}^{\beta} p_{\beta}^{\sigma}+2 \xi^{\prime} \bar{a}_{\sigma}^{i} \frac{d a_{\alpha}^{\sigma}}{d x} \\
& \xi^{\prime 2} \bar{q}_{\alpha}^{i}=\bar{a}_{\sigma}^{i} a_{\alpha}^{\beta} q_{\beta}^{\sigma}+\bar{a}_{\sigma}^{i} p_{\beta}^{\sigma} \frac{d a_{\alpha}^{\beta}}{d x}+\bar{a}_{\sigma}^{i} \frac{d^{2} a_{\alpha}^{\sigma}}{d x^{2}}
\end{aligned}
$$


or if they are multiplied by $a_{i}^{s}$,

$$
\begin{aligned}
& \xi^{\prime 2} a_{\sigma}^{s} \bar{p}_{\alpha}^{\sigma}=\xi^{\prime \prime} a_{\alpha}^{8}+\xi^{\prime} a_{\alpha}^{\beta} p_{\beta}^{8}+2 \xi^{\prime} \frac{d a_{\alpha}^{*}}{d x}, \\
& \xi^{\prime 2} a_{\sigma}^{8} \bar{q}_{\alpha}^{\sigma}=a_{\alpha}^{\beta} q_{\beta}^{s}+p_{\beta}^{8} \frac{d a_{\alpha}^{\beta}}{d x}+\frac{d^{2} a_{\alpha}^{8}}{d x^{2}} .
\end{aligned}
$$

These equations give the relations between the coefficients of the equations $(1: 1)$ and $(2: 6)$ subject to transformation $G(1: 2)$.

From these equations and those obtained by differentiating (2:9) the derivatives of the $a_{r}^{s}$ may be eliminated, obtaining

$$
\begin{aligned}
\xi^{\prime 3}\left(\frac{d \bar{p}_{\alpha}^{s}}{d \bar{x}}+\frac{1}{2} \bar{p}_{\alpha}^{\sigma} \bar{p}_{\sigma}^{s}-2 \bar{q}_{\alpha}^{s}\right)= & \xi^{\prime}\left(\frac{d p_{\beta}^{t}}{d x}+\frac{1}{2} p_{\rho}^{t} p_{\beta}^{\rho}-2 q_{\beta}^{t}\right) a_{\alpha}^{\beta} \bar{a}_{t}^{s} \\
& +\delta_{\alpha}^{s}\left[\frac{\xi^{\prime \prime \prime}}{\xi^{\prime}}-\frac{3}{2}\left(\frac{\xi^{\prime \prime}}{\xi^{\prime}}\right)^{2}\right] \xi^{\prime}
\end{aligned}
$$

By setting $s=\alpha$ and writing

$$
p \equiv \frac{1}{n}\left(\frac{d p_{\alpha}^{\alpha}}{d x}+\frac{1}{2} p_{\alpha}^{\beta} p_{\beta}^{\alpha}-2 q_{\alpha}^{\alpha}\right)
$$

and a like equation for $\bar{p}$ in the barred letters,

$$
\xi^{\prime 2} \bar{p}-p=\frac{\xi^{\prime \prime \prime}}{\xi^{\prime}}-\frac{3}{2}\left(\frac{\xi^{\prime \prime}}{\xi^{\prime}}\right)^{2} .
$$

The right member of this equation is commonly called the Schwartzian derivative. When this result is substituted in $(2: 11)$ with $\pi_{\alpha}^{*}$ defined by the equations

$$
2 \pi_{\alpha}^{\prime} \equiv \frac{d p_{\alpha}^{\prime}}{d x}+\frac{1}{2} \underset{p_{\alpha} p_{\sigma}^{\prime}}{\sigma}-2 \dot{q_{\alpha}}-\delta_{\alpha}^{\circ} p,
$$

and $\bar{\pi}_{a}^{:}$defined by like equations, we have

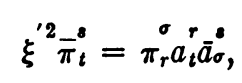

or the equivalent equations

$$
\xi^{\prime 2} a_{\sigma}^{s} \bar{\pi}_{t}^{\sigma}=\pi_{\sigma}^{*} a_{t}^{\sigma}
$$

The $\pi_{r}^{s}$ are accordingly the components of a mixed relative tensor. From $(2: 14)$ it follows immediately that the simplest invariant 


$$
\underset{\pi_{\alpha}}{\alpha} \equiv 0 \text {. }
$$

From $(2: 16)$, however, we obtain an invariant

$$
\pi \equiv\left|\pi_{r}^{8}\right|
$$

transforming by the equation

$$
\xi^{\prime 2 n} \bar{\pi}=\pi
$$

The functions $\xi^{\prime \prime \prime}$ and $\xi^{\prime \prime}$ can be eliminated from $(2: 18)$ and $(2: 13)$ yielding

$$
\xi^{\prime} \bar{\rho}=\rho,
$$

where

$$
\rho \equiv(1+4 n)\left(\frac{1}{\pi} \frac{d \pi}{d x}\right)^{2}+4 n \frac{1}{\pi} \frac{d^{2} \pi}{d x^{2}}+8 n^{2} p .
$$

is a relative invariant of weight two.

Eliminating $\xi^{\prime}$ from $(2: 19)$

$$
\bar{\sigma}=\sigma, \sigma=\rho / \pi^{1 / n} .
$$

To derive another tensor differentiate $(2: 16)$ and eliminate the derivatives of the $a_{r}^{s}$ by $(2: 9)$ and we have

$$
\begin{gathered}
\xi^{\prime 3}\left(\frac{d}{d \bar{x}} \bar{\pi}_{t}^{\beta}+\frac{1}{2} \tilde{p}_{\rho}^{\beta} \bar{\pi}_{t}^{\rho}-\frac{1}{2} \bar{p}_{t}^{\rho} \pi_{\rho}^{\beta}\right) a_{\beta}^{s}+2 \xi^{\prime} \xi^{\prime \prime} a_{\sigma}^{s} \bar{\pi}_{t}^{\sigma} \\
=\left(\frac{d}{d x} \pi_{\beta}^{s}+\frac{1}{2} p_{\sigma}^{s} \pi_{\beta}^{\sigma}-\frac{1}{2} p_{\beta}^{\sigma} \pi_{\sigma}^{s}\right) a_{t}^{\beta} .
\end{gathered}
$$

By differentiating $(2: 18)$ assuming $\pi \not \equiv 0$,

$$
\xi^{\prime} \xi^{\prime \prime}=\frac{1}{2 n}\left(\xi^{\prime 2} \frac{d \log \pi}{d x}-\xi^{\prime 3} \frac{d \log \bar{\pi}}{d \bar{x}}\right) .
$$

Substituting this in $(2: 22)$ and writing

$$
\pi_{t}^{\prime \beta} \equiv \frac{d}{d x} \pi_{t}^{\beta}+\frac{1}{2} p_{\rho}^{\beta} \pi_{t}^{\rho}-\frac{1}{2} p_{t}^{\rho} \pi_{\rho}^{\beta}-\frac{1}{n} \frac{d}{d x} \log \pi \cdot \pi_{t}^{\beta}
$$

we find

$$
\xi^{\prime 3} \pi_{t}^{\prime \beta} a_{\beta}^{8}=\pi_{\beta}^{18} a_{t}^{\beta}
$$

A generalization of this process will be called tensor differentiation. 
TENSOR DIFFERENTIATION

3. The function $\pi_{i}^{\prime} ;$ will be called the tensor derivative of $\pi_{i}$. It is a generalization of the derivative that is a tensor.

In general, if we have a mixed tensor of weight $k$ transforming as

$$
\xi^{k} a_{\alpha}^{i \alpha} s_{j}^{\alpha}=s_{\alpha}^{i} a_{j}^{\alpha},
$$

we may differentiate with respect to $x$ and eliminate the $d a_{r} / d x$ so introduced by $(2: 9)$, and the $\xi^{\prime \prime}$ by $(2: 23)$, obtaining

$$
\left(\xi^{\prime}\right)^{k+1} a_{\alpha}^{i} S_{j}^{\prime \alpha}=a_{j}^{\alpha} s_{\alpha}^{\prime i}
$$

where

$$
{s_{\alpha}^{\prime i}}^{i} \equiv \frac{d s_{\alpha}^{i}}{d x}+\Gamma_{\sigma s_{\alpha}}^{i \sigma}-\Gamma_{\alpha}^{\sigma i} s_{\sigma}^{i}-\frac{k}{2 n} s_{\alpha}^{i} \frac{d \log \pi}{d x}
$$

in any coördinates, and

$$
\Gamma_{i}^{i} \equiv \frac{1}{2} p_{i}^{i}+\frac{1}{4 n} \delta_{j}^{i} \frac{d \log \pi}{d x} .
$$

Consider the invariant which transforms by the equation

$$
\xi^{\prime k} I=I \text {. }
$$

When this is differentiated and $\xi^{\prime \prime}$ eliminated by $(2: 19)$, an invariant of weight $k+1$ is obtained.

This invariant,

$$
I^{\prime} \equiv \frac{d I}{d x}-\frac{k}{2 n} \cdot I \frac{d \log \pi}{d x},
$$

we call the invariant derivative of the invariant $I$. Likewise for the covariant vector $v_{i}$ of weight $k$ the vector derivative is

$$
v_{i}^{\prime} \equiv \frac{d v_{i}}{d x}-\Gamma_{i}^{o} v_{\sigma}-\frac{k}{2 n} v_{i} \frac{d \log \pi}{d x}
$$

and it is of weight $k+1$.

Similarly for the contravariant vector $v^{i}$ of weight $k$ the vector derivative is

$$
v^{i} \equiv \frac{d v^{i}}{d x}+\Gamma_{\rho}^{i} v^{p}-\frac{k}{2 n} v^{i} \frac{d \log \pi}{d x}
$$

and it transforms by the equations for a contravariant vector of weight $k+1$ 


$$
\left(\xi^{\prime}\right)^{k+1} \tilde{v}^{\prime \alpha} a_{\alpha}^{i}=v^{\prime i}
$$

Another set of contravariant vectors may be obtained from the results of equations $(2: 5)$ by noting that the derivation of this tensor depended only on the tensor equation (1:3), so if $\lambda^{i}$ is any contravariant tensor of weight zero,

$$
\Lambda^{i} \equiv \frac{d^{2} \lambda^{i}}{d x^{2}}+p_{\alpha}^{i} \frac{d \lambda^{\alpha}}{d x}+q_{\alpha}^{i} \lambda^{\alpha}
$$

is a contravariant tensor of weight two.

Two absolute tensors may be derived from the tensors $\pi_{r}^{s}$ and $\pi_{r}^{\prime s}$ by the use of $(2: 18)$. These will be represented by

$$
\begin{aligned}
& P_{r}^{s} \equiv \pi_{s}^{r} / \pi^{1 / n}, \\
& Q_{r}^{s} \equiv \pi_{r}^{\prime s} / \pi^{3 /(2 n)} .
\end{aligned}
$$

Since by $(3: 4)$

$$
\pi^{\prime} \equiv 0,
$$

$\pi$ acts as a constant in tensor differentiation, and

$$
P_{r}^{\prime}=\pi_{r}^{\prime} / \pi^{1 / n}
$$

and

$$
Q_{r}^{8}=P_{r}^{\prime s} / \pi^{1 /(2 n)}
$$

By a change of independent variable (4.2) the absolute tensors $P_{r}^{s}$ and $Q_{r}^{s}$ are identified with the relative tensors $\pi_{r}^{s}$ and $\pi_{r}^{\prime s}$.

In $\S 6$ we argue that if $(2: 10)$ is an independent set of equations, $(2: 11)$ and thence $(2: 13)$ and $(2: 16)$ consisting of $n^{2}+1$ equations are equivalent to the $n^{2}$ equations $(2: 10)$. Hence the $n^{2}$ equations $(2: 16)$ have one identity among them and (2:17) is that identity.

Other tensor identities will be listed here. From (3:8) it follows that

$$
\left|P_{r}^{*}\right|=1
$$

and from $(3: 8)$ and $(2: 17)$ that

$$
P_{\rho}^{\rho}=0
$$

since from $(2: 24)$ and $(2: 17)$

$$
\pi_{p}^{\prime p}=0
$$


it follows from $(3: 8)$ that

$$
Q_{\rho}^{\rho}=0
$$

Another identity may be obtained by differentiating $\pi \equiv\left|\pi_{r}^{s}\right|$,

$$
\pi \frac{d}{d x} \log \pi=\frac{\partial \pi}{\partial \pi_{\rho}^{\sigma}} \frac{d}{d x} \pi_{\rho}^{\sigma} ;
$$

also

$$
\frac{\partial \pi}{\partial \pi_{\rho}^{\sigma}} \pi_{\rho}^{\sigma}=n \pi
$$

Substituting the value of $\pi$ from $(3: 16)$ in $(3: 15)$, and introducing the tensor of weight -2

$$
\begin{gathered}
\Sigma_{s}^{r} \equiv \frac{\partial \pi}{\partial \pi_{r}^{s}}, \\
\Sigma_{\rho}^{\sigma}\left(\frac{d}{d x} \pi_{\sigma}^{\rho}-\frac{1}{n} \pi_{\sigma}^{\rho} \frac{d}{d x} \log \pi\right)=0 .
\end{gathered}
$$

In the next article a system of coördinates will be introduced in which tensor derivatives and ordinary derivatives are identical, reducing the above equation to

$$
\Sigma_{\rho}^{\sigma} \pi_{\sigma}^{\prime \rho}=0,
$$

which, being a tensor equation, is valid in all coördinates. The same result can be obtained here by using $(3: 2.1)$.

\section{SEMICANONICAL FORM}

4. A system of coördinates exists for which tensor differentiation and ordinary differentiation are identical. It follows from the equation $(2: 18)$ $\xi^{\prime 2 n} \bar{\pi}=\pi$ that by choosing

$$
\xi^{\prime}=\pi^{1 /(2 n)}
$$

there results

$$
\bar{\pi}=1, \frac{d \bar{\pi}}{d x}=0
$$

Since $\bar{\pi}$ is an invariant for a change of dependent variable a new choice of dependent variable does not alter $(4: 2)$. By reference to equations $(1: 8)$ it is apparent that the $p_{r}^{s}$ will vanish if a transformation is made with $a_{r}^{s}$ satisfying 


$$
2 \frac{d a_{r}^{s}}{d x}+\stackrel{\alpha}{a_{r} p_{\alpha}^{s}}=0 .
$$

From the existence theorems for equations of this type we know that there exist $n$ solutions $a_{r}^{0 s}, s=1, \cdots, n$. Other sets are given by $c_{r}^{\alpha} a_{\alpha}^{0 s}$, the $c_{r}^{\alpha}$ being arbitrary constants. For the solutions $a_{r}^{0 s}$ equations $(1: 3)$ are

$$
y^{s}=a_{\alpha}^{0 s} \bar{y}^{\alpha} .
$$

The coördinates $\bar{y}^{\alpha}$ may be subjected to a projective transformation

$$
\bar{y}^{\alpha}=c_{\beta}^{\alpha} \bar{y}^{\beta} .
$$

Then

$$
y^{2}=\stackrel{\alpha}{c_{\dot{\beta}} a_{\alpha}^{0 i}} \stackrel{\bar{y}}{\beta},^{\beta}
$$

so the coördinates in which the $p$ 's vanish are determined to within a general linear homogeneous transformation.

The formulas for tensor differentiation indicate that in these coördinates tensor derivatives are ordinary derivatives. An important consequence of this is that the formulas for differentiation, notably for the differentiation of products and quotients, are valid for tensor differentiation. For if any product, or product and quotient, which is a tensor, is differentiated in these coördinates, the same formula will hold if tensor differentiation is substituted for ordinary differentiation. The resulting formula will be of the same tensor character except that the weight will have been increased by one. But from the fundamental properties of tensors, tensor equations holding for one system of coördinates are valid in all.

5. Let us consider a transformation on the parameter

$$
\bar{x} \equiv \xi(x),
$$

where $\xi$ satisfies the differential equation

$$
\frac{\xi^{\prime \prime \prime}}{\xi^{\prime}}-\frac{3}{2}\left(\frac{\xi^{\prime \prime}}{\xi^{\prime}}\right)^{2}+p=0,
$$

and $p$ is defined by (2:12). From (2:13) it follows that for the new parameter

$$
\bar{p}=0 .
$$

Since $\bar{p}$ is invariant for transformations on the dependent variables $((2: 13)$ or $(1: 13))$ the semicanonical transformation (4:3.1) transforms $(1: 1)$ to 


$$
\frac{d^{2} y^{i}}{d x^{2}}+q_{\alpha}^{i} y^{\alpha}=0, q_{\alpha}^{\alpha}=0, \pi_{r}^{8}=-q_{r}^{8}
$$

Any transformation that leaves invariant the equations $p_{r}^{s}=0$ will be determined by $(2: 7)$ :

$$
\delta_{j}^{i} \xi^{\prime \prime}+2 \xi^{\prime} \bar{a}_{\sigma}^{i} \frac{d a_{j}^{\sigma}}{d x}=0
$$

Solving for the derivatives $d a_{j}^{i} / d x$ and integrating,

$$
a_{j}^{i}=c_{j}^{i}\left(\xi^{\prime}\right)^{-1 / 2}
$$

If further $q_{\alpha}^{\alpha}$ is to remain equal to zero, in virtue of $(2: 8)$

$$
\bar{a}_{\sigma}^{\tau} \frac{d^{2} a_{\tau}^{\sigma}}{d x^{2}}=0
$$

Substituting (5:4) in this equation, the solution is given by

$$
\left(\xi^{\prime}\right)^{-1 / 2}=c x+d, \xi=\frac{a x+b}{c x+d}, a d-b c=1 .
$$

Hence canonical coördinates are related by these equations and

$$
a_{j}^{i}=(c x+d) c_{j}^{i}
$$

\section{Hence}

The necessary and sufficient condition that two systems of equations (1:1) be equivalent is that after each is reduced to the form (5:3) there exist constants satisfying

$$
(c x+d)^{-4} c_{p}^{s} \bar{\pi}_{r}^{p}=\pi_{p}^{s} c_{r}^{p}
$$

Equations (5:3) will be said to be in canonical form.

The EQUIVALENCE PROBLEM

6. To apply the theorem of the last section to test the equivalence of two systems of differential equations of the type $(1: 1)$ requires the integration of equations of the type $(4: 3)$ and $(5: 1)$. An algebraic test of equivalence will now be given. The necessary and sufficient test for equivalence is that there exist functions $\xi(x)$ and $a_{r}^{s}(x)$ satisfying (2:9) and (2:10). Equations (2:11) were obtained by solving for the $d a_{r}^{s} / d x$ and $d^{2} a_{r}^{s} / d x^{2}$ from (2:9) and substituting in $(2: 10)$, hence $(2: 10)$ can be obtained from $(2: 9)$ and $(2: 11)$. The equivalence conditions are now given by $(2: 9)$ and $(2: 11)$. Equation $(2: 13)$ 
is a linear combination of certain equations from the set $(2: 11)$. The set of equations $(2: 16)$ is obtained by eliminating the right member of $(2: 13)$ from $(2: 11)$ by means of $(2: 13)$. It follows that $(2: 11)$ may be obtained from $(2: 13)$ and $(2: 16)$ and the equivalence conditions are now (2:9), (2:13) and $(2: 16)$, and we seek functions $\xi^{\prime}, a_{r}^{s}$ satisfying these equations. We will first make the choice of the function $\xi^{\prime}$ to satisfy these equations. Eliminating the $a_{r}^{s}$ from $(2: 16)$ obtaining $(2: 18)$ it is clear that $\xi^{\prime}$ must be chosen to satisfy $(2: 18)$. But $(2: 13)$ also determines $\xi^{\prime}$. The condition that these are consistent is given by $(2: 21)$. Regarding now the $\xi^{\prime}$ as known functions in $(2: 9)$ and $(2: 16)$, the solution of the equivalence problem rests upon the determination of the existence of the solutions $a_{r}^{s}$ satisfying simultaneously these two sets and $\left|a_{r}^{s}\right| \neq 0$. By differentiating $(2: 16)$ the quantities $d a_{r}^{s} / d x$ appear which must be consistent with their values as given by (2:9). Equations $(2: 25)$ are a necessary condition for this and so are all analogous sets of equations obtained by tensor differentiation.

Consider this sequence of equations

$$
\begin{gathered}
\xi^{\prime} a_{\sigma}^{s} \bar{\pi}_{t}^{\sigma}=\pi_{\sigma}^{s} a_{t}^{\sigma}, \\
\xi^{\prime 3} a_{\sigma}^{s} \bar{\pi}_{t}^{\prime \sigma}=\pi_{\sigma}^{\prime s} a_{t}^{\prime}, \\
\xi^{\prime} a_{\sigma}^{s} \bar{\pi}_{t}^{\prime \prime \sigma}=\pi_{\sigma \sigma}^{\prime s},
\end{gathered}
$$

the accents representing tensor derivatives. The $a_{r}^{s}$ are the unknown functions and the sets of equations will either be inconsistent or yield solutions satisfying $\left|a_{r}^{s}\right|=0$, in which case the sets of differential equations of the type $(1: 1)$ will be not equivalent, or the first $m$ sets will yield a solution which will satisfy the $(m+1)$ th, and the inequality $\left|a_{r}^{s}\right| \neq 0$. Consider the $a_{r}^{s}$ satisfying the first $m$ sets of (6:1) and $\left|a_{r}^{s}\right| \neq 0$. The derivatives $d a_{r}^{s} / d x$ must satisfy (2:9). The only additional conditions not already satisfied are precisely that the $d a_{r}^{i} / d x$ satisfy the $(m+1)$ th set, and these conditions are satisfied by our hypothesis. Hence the following theorem:

The necessary and sufficient conditions that there exist $n^{2}+1$ functions $a_{r}^{s}, \xi$ which will transform one given differential system (1:1) into another given system $(2: 6)$ is that their invariants $\sigma(2: 21)$ be equal and that there exist a number $m$ such that a solution satisfying the first $m$ sets of (6:1) and $\left|a_{r}^{s}\right| \neq 0$ will satisfy the $(m+1)$ th set of $(6: 1)$.

We define a complete system of tensors as a system that determines the equations $(1: 1)$ to within a transformation under the group $G(1: 2)$. As an alternative of the above theorem we have the following: 
The tensor $\pi_{r}^{s}$ and its tensor derivatives in conjunction with the invariant $\sigma$ constitute a complete system of tensors.

INVARIANTS OF A MIXED TENSOR AND THE NORMAL FORM

7. The tensor $A_{r}^{s}$ is transformed by the equations

$$
\bar{A}_{r}^{s}=A_{\rho}^{\sigma} a_{r}^{\rho} \bar{a}_{\sigma}^{8}
$$

or in the matrix calculus

$$
\bar{A}=a^{-1} A a \text {. }
$$

It is known* that a coördinate system can be chosen so that the matrix $A$

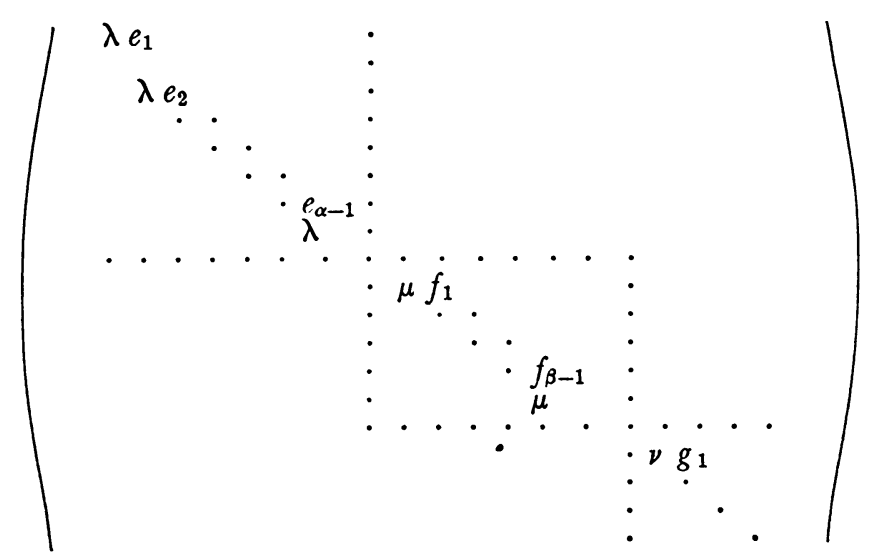

where the $\lambda$ 's, $\mu$ 's, $\nu$ 's, $\cdots$ are roots of multiplicities $\alpha, \beta, \gamma, \cdots$ of the characteristic equation $\Delta(\lambda)=0$ and the $e$ 's, $f$ 's, $g$ 's, . . are 0 's or 1's. The roots $\lambda_{i}$, including the $\alpha \lambda$ 's, the $\beta \mu$ 's, ‥ of (7:2), are invariants of the tensor $A_{r}^{s}$, but unless they are all distinct they do not determine it. Matrices $A_{r}^{s}$ and $\bar{A}_{r}^{s}$ satisfying $(7: 1)$ are called equivalent. It is a known theorem that the criterion of equivalence is that the $\lambda$ matrices of the two matrices have the same invariant factors.

The tensor $A_{r}^{s}$ has the following invariants:

$$
A_{\rho}^{\rho}, A_{\rho}^{\sigma} A_{\sigma}^{\rho}, \cdots, A_{\sigma_{1}}^{\rho} A_{\sigma_{2}}^{\sigma_{1}} \cdots A_{\rho}^{\sigma_{n-1}} .
$$

When the tensor has been reduced to the normal form $(7: 2)$ these become

$$
\Sigma \lambda_{i}, \Sigma\left(\lambda_{i}\right)^{2}, \cdots, \Sigma\left(\lambda_{i}\right)^{n} .
$$

It is well known that this set of symmetric functions is equivalent to the ele-

* Jordan, Traité des Substitutions, 1870. Dickson, Modern Algebraic Theories, Chapter V. 
mentary symmetric functions and hence determines the roots $\lambda_{i}$. The invariants $(7: 3)$ constitute, therefore, a set of rational invariants that is equivalent to the set $\lambda_{1}, \cdots, \lambda_{n}$.

The matrix $(7: 2)$ is arranged in blocks along the principal diagonal. If this matrix is used to define a representation

$$
\eta^{i}=A_{\alpha}^{i} y^{\alpha}
$$

vectors in the subspace $y^{1}, \cdots, y^{\alpha}$ or $y^{\alpha+1}, \cdots, y^{\alpha+\beta}$, etc., are transformed into vectors in the subspace. Such subspaces have been called invariant subspaces.*

The subspaces are further decomposable if some of the $e$ 's, $f$ 's, $\cdots$ are zero. If, for example, two numbers $e_{1}$ and $e_{2}$ are zero, the subspace $y^{1}, y^{2}$ is invariant in a more special sense. No vector in this space is changed in direction by the representation. In case the corresponding $\lambda$ is equal to unity the dimensionality of the transformation is reduced (in this case by 2 ).

For the tensor $A_{r}^{s} \equiv \pi_{r}$ the $n$ algebraic invariants $\lambda_{1}, \cdots, \lambda_{n}$ are subject to the condition that their sum is zero because of the identity $(2: 17)$. The invariant $\sigma(2: 21)$ brings the number of algebraic invariants up to $n$, plus their tensor derivatives.

\section{NORMAL COORDINATES}

When the differential equations are reduced to the canonical form, then with a fixed $x_{0}$ transformations of the dependent variable (of the type $(4: 4)$ ) will reduce the canonical form to a form $d^{2} y^{i} / d x^{2}=\pi_{\alpha}^{i} y^{\alpha}$ with matrix given by $(7: 2)$. In general the new coördinates are valid only in the neighborhood of $x_{0}$. We shall designate such a reference system as normal coördinates.

\section{A DYNAMICAL INTERPRETATION}

8. From established existence theorems it is known that through any point $y^{i}$ in a direction determined by the $n$ derivatives $d y^{i} / d x$ there exists an integral curve with parameter $x_{0}$ at $y^{i}$.

If the independent variable is interpreted as time the equations $(1: 1)$ define the motion of a particle. The coördinates may be interpreted as Cartesian, and under the group $G$ all axes rotating in an arbitrary relative fashion become equivalent. The time is ordered in any continuous manner. We are interested in finding properties that are identifiable in any coördinate system, the coördinate system comprising $n$ dimensions in space and one in time.

* Weyl, Gruppentheorie und Quantenmechanik, Chapter I. 
The existence theorem in dynamical terms states that the motion is determined by the initial point, velocities and time, and that there exist $2 n$ independent solutions $\left(y_{\alpha_{1}}^{1}, \cdots, y_{\alpha_{1}}^{n}\right), \alpha=1, \cdots, 2 n$. A general solution is given by a linear combination of the fundamental set. For fixed initial conditions

$$
\begin{aligned}
& y^{i}\left(x_{0}\right)=c^{\alpha} y_{\alpha \mid}^{i}\left(x_{0}\right), \\
& \left(\frac{d y^{i}}{d x}\right)_{x_{0}}=c^{\alpha}\left(\frac{d y_{\alpha \mid}^{i}}{d x}\right)_{x_{0}}, \alpha \text { summed from } 1, \cdots, 2 n,
\end{aligned}
$$

the constants $c^{\alpha}$ are determined. A curve and its parameterization is thus determined. A new choice of parameter $x_{0}$ is not a transformation of parameter but determines a new curve through the same point in the same direction. This is apparent when it is noticed that the differential equations (1:1) are not transformed but the acceleration has been changed by the new values of the parameter in the functions $p_{r}^{s}(x)$ and $q_{r}^{s}(x)$.

If $\lambda$ is defined to be a relative invariant of weight $2, \pi-\lambda \delta_{r}^{s}$ will be a tensor. If $h^{i}$ are defined to be components of a tensor of weight $k$ the equations

$$
\lambda(x) h^{i}=\pi_{\alpha}^{i} h^{\alpha}
$$

will be invariant under $G$. Since the theory of $\$ 6$ which made use of transformations on the dependent variable only will apply here we can find $n$ roots $\lambda_{1}, \cdots, \lambda_{n}$ which, when substituted in $(8: 1)$, determine an invariant direction for a simple root or an invariant $r$-space for an $r$-fold root. These directions are associated with a point on the integral curve, for they are functions of the parameter of the point and are invariant under a transformation of parameter.

Normal coördinates reducing $(1: 1)$ to

$$
\frac{d^{2} y^{i}}{d x^{2}}=\pi_{\alpha}^{i} y^{\alpha},
$$

where the $\pi_{r}^{s}$ have the matrix $(7: 2)$, are valid at the point with parameter $x$. The $h^{i}$ of equations $(8: 1)$ are coördinates of a point referred to axes parallel to the coördinates axes and origin at the point $y^{i}$ on the integral curve $C$. Since the same transformations which determine the coördinates as along the invariant directions defined by $(8: 1)$ will transform $(8: 2)$ to a form with matrix $(7: 2)$ valid in the neighborhood of the point $x$ we may conclude that a point moving in the invariant direction $h^{i}(\lambda)$ at $y^{i}$ will be accelerated in its direction of motion. 
Our interpretation applies only in the special coördinates but the directions are uniquely defined in all. Hence the theorem:

There exist at each point of an integral curve of (1:1) uniquely determined directions $\lambda_{1}, \cdots, \lambda_{n}$. A coördinate system exists (under $G$ ) such that in the direction determined by a simple root $\lambda_{i}$ there exists a motion for which the acceleration and velocity vectors have this same direction. In the case of an r-fold root there exists a corresponding $r$-space in which the motion in any direction is accelerated in a direction lying in the r-space. If further $k(=0,1, \cdots, r-1)$ of the $e_{1}, \cdots, e_{r-1}$ are zero there exists a $(k+1)$-space in the r-space in which motion in any direction is accelerated in that direction.

A special case of interest is given by

$$
\pi_{r}^{*}=\text { const. }
$$

in canonical coördinates. In this case the reduction to normal coördinates is valid for a finite region.

Also a consequence of $(8: 3.1)$ is that

$$
\pi=\text { const. in canonical coördinates }
$$

and

$$
\pi_{r}^{\prime s}=0 \text { in any coördinates. }
$$

Conversely if $(8: 3.3)$ is assumed to hold in a coördinate system it will obtain in canonical coördinates and

$$
\bar{\pi}_{r}^{\prime \prime} \equiv \frac{d \bar{\pi}_{r}^{0}}{d x}-\frac{1}{n} \bar{\pi}_{r}^{\prime} \frac{d}{d x} \log \bar{\pi}=0, \bar{\pi}_{r}^{*}=-\bar{q}_{r}^{*}
$$

or

$$
\frac{d \dot{q}_{r}^{*}}{d x}=\frac{1}{n} \dot{q}_{r}^{\prime} \frac{d}{d x} \log \bar{\pi}, \quad \tilde{q}_{a}^{\alpha}=0
$$

Integrating

$$
\bar{q}_{r}^{*}=\bar{\pi}^{1 / n} c_{r}^{*}, c_{\alpha}^{\alpha}=0,\left|\dot{c}_{r}^{*}\right|=1 .
$$

The function $\bar{\pi}$ will not in general be a constant in these coördinates and the transformation on the independent variable has been utilized in $(5: 1)$. We seek the condition that $\bar{\pi}$ be a constant in canonical coördinates.

By reference to $(2: 18)$ it appears therefore that for $\bar{\pi}$ to be constant in canonical coördinates

$$
\xi^{\prime}=c \pi^{1 /(2 n)} .
$$


Since $\xi^{\prime}$ has been chosen to satisfy $(5: 1), \pi$ must satisfy

$$
(4 n+1)\left(\frac{d \pi}{d x}\right)^{2}-4 n \pi \frac{d^{2} \pi}{d x^{2}}-8 n^{2} p=0, \pi \neq 0 .
$$

This is the invariant $\pi^{2} \rho$ of equation $(2: 20)$.

Since $\pi \neq 0$ equation $(8: 8)$ is equivalent to $\rho=0$. If then we assume $\rho=0$ in any coördinate system we know that $\pi^{1 /(2 n)}$ satisfies $(5: 1)$. To transform to canonical coördinates it is only necessary to take $\xi^{\prime}$ as a solution of $(5: 1)$ so we may take $\xi^{\prime}=\pi^{1 /(2 n)}$. Then in canonical coördinates we will have $\bar{\pi}=1$. Hence from $(8: 4)$ it follows that $\bar{\pi}_{r}^{s}=$ const. and we have the following theorem:

The necessary and sufficient conditions that the set of algebraic invariants $\lambda_{1}, \cdots, \lambda_{n}$ will be reducible to constants by a change of the independent variable are that

$$
\pi_{r}^{\prime s}=0, \text { and } \rho=0 .
$$

Under the condition $\rho=0$ we showed above that canonical coördinates could be introduced such that $\bar{\pi}=1$. Conversely from the conditions $\pi=1$ and $\rho=0$ it follows that $p=0$. Hence:

The vanishing of the invariant $\rho$ is the necessary and sufficient condition for the existence of a coördinate system that is simultaneously semicanonical and canonical.

Since $(8: 8.1)$ insures that $(8: 3.1)$ is valid in canonical coördinates, they are the necessary and sufficient conditions for the existence of a coördinate system which is, simultaneously, normal for a finite region, semicanonical and canonical.

The invariant equation

$$
\pi=0
$$

may be interpreted by transforming to canonical coördinates where it implies a relation between the accelerations.

The tensor equations

$$
\pi_{r}^{*}=0
$$

in any coördinates imply that in canonical coördinates

$$
\frac{d^{2} y^{i}}{d x^{2}}=0, y^{i}=a^{i} x+b^{i},
$$

and are therefore the invariant conditions insuring the possibility of transforming the integral curves to straight lines. 


\section{The METRIC}

9. Consider a set of $n$ independent solutions of (8:1) for $\lambda=\lambda_{1}, \cdots, \lambda_{n}$ roots of the characteristic equation, and designate these by $\lambda_{j 1}^{i}$, a set of tensors of weight zero. The index $j \mid$ designates the vector, and $i$ the components.

We define

$$
g^{\mu \nu}=\lambda_{\alpha \mid}^{\mu} \lambda_{\alpha \mid}^{\prime},
$$

and proceed to develop other formulas familiar in the calculus of Ricci and Levi-Cevita.

Defining $g_{\mu \nu}$ as the cofactor of $g^{\mu \nu}$ divided by $\left|g^{r s}\right|$, and multiplying (9:1) by $g_{\mu \rho}$,

$$
g_{\mu \rho} \lambda_{\alpha \mid}^{\mu} \lambda_{\alpha \mid}^{\nu}=\delta_{\rho}^{\nu} .
$$

Let $\lambda_{\alpha \mid \mu}$ be the cofactor of $\lambda_{\alpha \mid}^{\mu}$ divided by $\left|\lambda_{\alpha \mid}^{i}\right|$; multiplying $(9: 2)$ by $\lambda_{\beta \mid \nu}$,

$$
\lambda_{\beta \mid \nu}=g_{\mu \rho} \lambda_{\beta \mid}^{\mu} \text {. }
$$

Multiplying (9:1) by $\lambda_{\beta \mid \nu}$,

$$
\lambda_{\beta \mid}^{\mu}=g^{\mu \nu} \lambda_{\beta \mid \nu} .
$$

Equations $(9: 3)$ and $(9: 4)$ exhibit the technique of raising and lowering indices.

Multiplying (9:3) by $\lambda_{\beta \mid \nu}$,

$$
g_{\mu \nu}=\lambda_{\alpha \mid \mu} \lambda_{\alpha \mid \nu}
$$

The functions $g_{\mu \nu}(x)$ will be taken for a metric on an integral curve.

Since

$$
g_{\mu \nu} \lambda_{\alpha \mid}^{\mu} \lambda_{\beta \mid}^{\nu}=\delta_{\alpha}^{\beta},
$$

the vectors $\lambda_{j \mid}$ constitute a set of orthogonal unit vectors.

We are now able to measure angles and define the angle between the unit vectors $u^{i}$ and $v^{i}$ as

$$
\cos \theta=g_{i j} u^{i} v^{j}
$$

If $\mu^{i}$ is a unit vector, the angles with the coördinate axes are given by

$$
c_{\alpha \mid} \equiv g_{i j} \mu^{i} \lambda_{\alpha \mid}^{j} \quad(\alpha=1, \cdots, n) .
$$

The magnitude $\mu$ of a vector $\mu^{i}$ will be defined by the invariant

$$
\mu^{2}=g_{i j} \mu^{i} \mu^{j}
$$


The coördinates so defined by the vectors $\lambda_{j l}^{i}$ will be called local coördinates. They enable measurements of angles and vectors to be made at a point, but as yet our geometry does not permit a comparison at neighboring points.

\section{PARALLEL VECTORS}

10. At any point $P\left(x_{0}\right)$ on a curve a coördinate system and metric will be established as in the last article. Parallel coördinate systems will be determined by displacing the given $n$-hedron along the curve. The $\alpha$ axis of the $n$-hedron is given by the vector $\lambda_{\alpha \mid}^{i}\left(x_{0}\right)$. The new parallel $\lambda_{\alpha \mid}^{i}(x+d x)$ will have an increment computed by the invariant formula $\lambda_{\alpha \mid}^{\prime i}=0$. It follows that for the metric so defined

$$
g_{i j}^{\prime}=0 \text {. }
$$

The coördinate systems uniquely established in this manner will be called parallel coördinates. In general, a vector $\mu^{i}$ will be said to be parallelly displaced if $\mu^{\prime i}=0$.

Consider two vectors $\lambda^{i}$ and $\mu^{i}$ which are displaced parallelly. The change of the angle between them after parallel displacement when referred to parallel coördinates will be $((3: 4), k=0)$

$$
\frac{d}{d x}\left(g_{i j} \lambda^{i} \mu^{j}\right)=\left(g_{i j} \lambda^{i} \mu\right)^{\prime}=0 .
$$

Hence:

Angles measured in parallel coördinates are preserved by parallel displacement.

At the point $P\left(x_{0}\right)$ the local coördinates and parallel coördinates were identical. If the vectors of local coördinates are represented by $l_{\alpha 1}^{i}$,

$$
l_{\alpha \mid}^{i}\left(x_{0}\right)=\lambda_{\alpha \mid}^{i}\left(x_{0}\right) \text {. }
$$

The twist of local coördinates may be measured by

$$
\begin{aligned}
T & =\frac{d}{d x}\left(g_{i j} \lambda_{\alpha \mid}^{i} l_{\beta}^{j}\right)=\left(\lambda_{\alpha \mid j} l_{\beta \mid}^{j}\right)^{\prime}=\lambda_{\alpha \mid j} l_{\beta \mid}^{\prime i}, \\
T & =l_{\alpha \mid j} l_{\beta}^{\prime j} .
\end{aligned}
$$

UNIVERSITY OF WASHINGTON, SeAtTLE, Wash. 